\title{
CLIC PROGRESS TOWARDS MULTI-TEV LINEAR COLLIDERS
}

\author{
Hans-Heinrich Braun for the CLIC team, CERN, Geneva, Switzerland
}

\begin{abstract}
New parameters of an $\mathrm{e}^{+} / \mathrm{e}^{-}$Linear Collider based on CLIC technology for a luminosity of $7 \cdot 10^{34} \mathrm{~cm}^{-2} \mathrm{~s}^{-1}$ at a nominal energy of $3 \mathrm{TeV}$ are presented. They are derived in part from the very successful tests and experience accumulated in the CLIC Test facility, CTF2. A new and ambitious test facility, CTF3, presently under construction at CERN in an international collaboration of laboratories and institutes, and aimed at demonstrating the key feasibility issues of the CLIC scheme, is described.
\end{abstract}

\section{INTRODUCTION}

The next energy frontier in High Energy Physics is on the $\mathrm{TeV}$ scale, which will first be explored by the LHC. Just as $\mathrm{e}^{+} / \mathrm{e}^{-}$colliders provided an essential complement to hadron-hadron colliders in the $100 \mathrm{GeV}$ energy range, establishing beyond doubt the validity of the Standard Model of particle physics, so we expect that higherenergy $\mathrm{e}^{+} / \mathrm{e}^{-}$colliders will unravel the $\mathrm{TeV}$ physics, to be unveiled by the LHC. They provide very clean experimental environments and democratic production of all particles within the accessible energy range, including those with only electroweak interactions [1]. One of the options for such an $\mathrm{e}^{+} / \mathrm{e}^{-}$collider is the Compact LInear Collider CLIC with the unique feature of a centre of mass energy as high as $3 \mathrm{TeV}$.

The CLIC study together with its former test facility CTF2 and the present test facility CTF3 have been described in $[2,3]$. Here we show recent developments following the recommendations of the International Linear Collider Technical Review Committee (ILC-TRC) and a major revision of the CLIC design and parameters.

The ILC-TRC committee has performed in 2002 an indepth analysis of three linear collider technologies, namely NLC/JLC, TESLA and CLIC [4]. The results of this analysis were summarized in a ranked list of recommended $R \& D$ items for each technology. The rankings are defined as

- R1: R\&D needed for feasibility demonstration

- $\quad$ R2: R\&D needed to finalize design choices

- R3: R\&D needed before starting production

- R4: R\&D desirable for technical/cost optimization

Following these recommendations the CLIC study team decided to focus resources on the CLIC specific R1 and $\mathrm{R} 2$ issues aiming to demonstrate key feasibility issues and to finalize design choices before 2010. The program of the present CLIC test facility (CTF3) has been aligned with these goals [5].

The ILC-TRC R1 and R2 issues specific for CLIC are

R1.1 Test of damped accelerating structure at design gradient and pulse length

R1.2 Validation of drive beam generation scheme with fully loaded linac operation
R1.3 Design and test of damped ON/OFF power extraction and transfer structure (PETS)

R2.1 Developments of structures with hard-breaking materials (W, Mo...)

R2.2 Validation of stability and losses of drive beam decelerator; design of machine protection system

$R 2.3$ Test of relevant linac sub-unit with beam

R2.4 Validation of drive beam $40 \mathrm{MW}, 937 \mathrm{MHz}$ MultiBeam Klystron with long RF pulse

R2.5 Effects of coherent synchrotron radiation (CSR) in bunch compressors

R2.6 Design of an extraction line for $3 \mathrm{TeV}$ c.m.

$R 1.1-R 1.3$ as well as $R 2.1-R 2.3$ are addressed in CTF3 and described in the following section. Studies have been performed [6] on the klystron issue R2.4. An experimental verification is presently considered less urgent, because an adaptation of the drive beam accelerator structures to a lower klystron power and a corresponding larger number of klystrons is straight forward. The coherent synchrotron radiation in the CLIC bunch compressors $R 2.5$ and the design of the extraction line $R 2.6$ are treated in work package 6 of the EUROTeV design study [7], which will be completed by end 2007 .

\section{CLIC FEASIBLILITY ISSUES AND CTF3}

Figure 1 shows an outline of CTF3 with indications, where in the facility the various feasibility issues are addressed. The drive beam injector and accelerator together with a novel chicane for longitudinal bunch length manipulations have been successfully constructed and commissioned in 2003 and 2004 with major contributions from INFN-LNF, SLAC, IN2P3-LAL, Northwestern University and Uppsala University $[8,9,10,11]$. A major achievement of the drive beam accelerator commissioning is the demonstration of fully loaded operation of the drive beam accelerator with nominal beam current of $3.5 \mathrm{~A}$. The RF to beam power transfer efficiency is more than $95 \%$. No indications of beam break-up were observed. This achievement is an important step for the validation of the CLIC drive beam scheme as requested as feasibility issue $R 1.2$ of the ILC-TRC.

A beam line with a special $30 \mathrm{GHz}$ power extraction structure to feed the new high gradient test stand has been commissioned in 2004 [12]. An overmoded low loss waveguide transfers the RF over a distance of $17 \mathrm{~m}$ from the extraction structure to the test stand in a neighbouring building. So far a peak power of more than $50 \mathrm{MW}$ in $70 \mathrm{~ns}$ long pulses has been achieved at the test stand. In the next CTF3 run, starting now, this new RF source will be used to test CLIC accelerating structures to address the ILC-TRC R1.1 and $R 2.1$. 


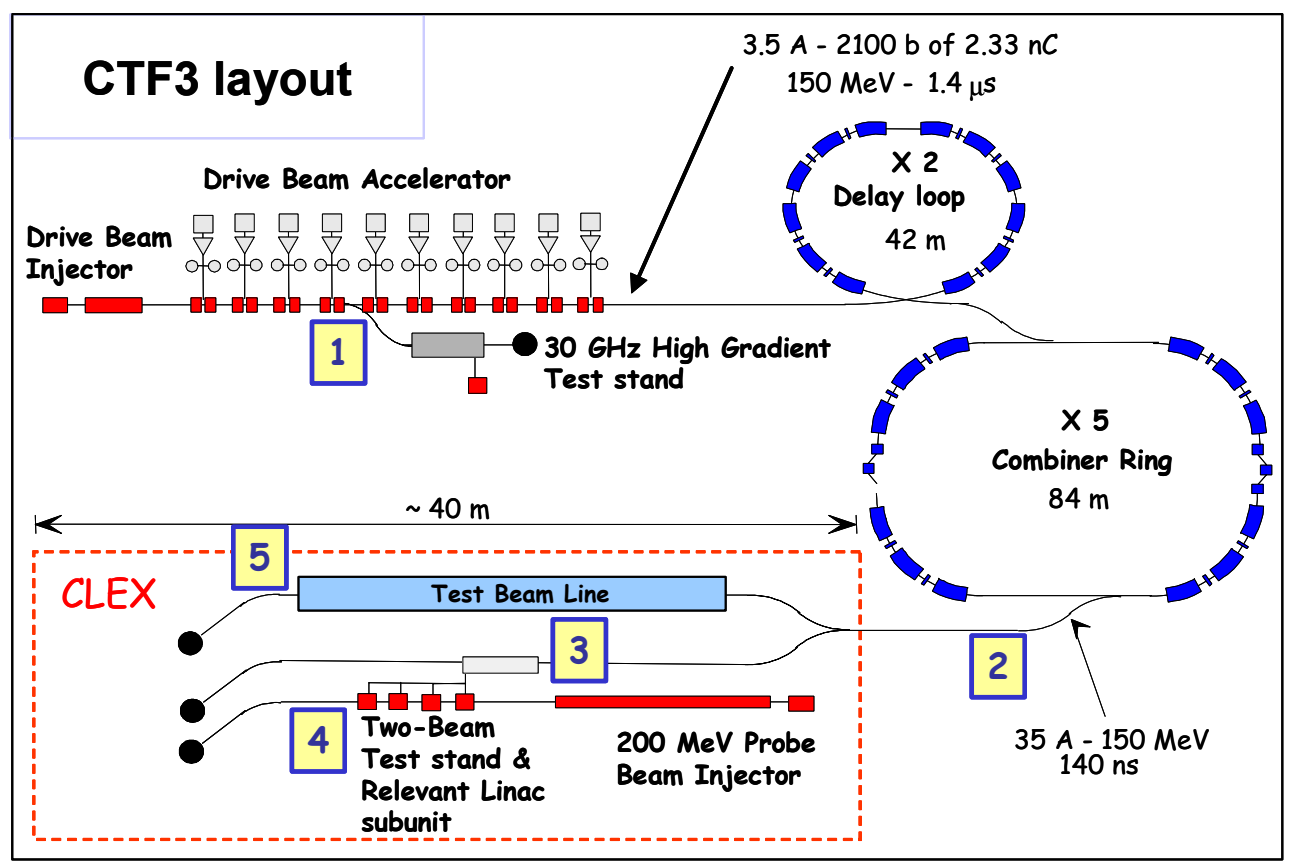

Figure 1:

Schematic layout of CTF3 with locations of the key feasibility tests. The test of a damped accelerating structure at the design gradient and pulse length $(R 1.1$ and $R 2.1)$ requires the linac-driven high-gradient test stand (location 1$)$. The validation of the drive-beam generation scheme with a fully-loaded linac $(R 1.2)$ requires the combiner ring (location 2$)$. Test of a power-extraction structure $(R 1.3$, location 3$)$ and the test of a relevant linac sub-unit with beam $(R 2.3$, location 4) require the CTF3 experimental area CLEX. CLEX consists of the probe beam injector, the two beam test stand and the Test Beam Line (TBL). The TBL is a scaled model of a CLIC decelerator sector and will validate beam stability and losses in the drive-beam decelerator as well as the design of an appropriate machine protection system $(R 2.2$, location $5)$.

The delay loop is designed and built by INFN-LNF. Commissioning will start in autumn this year. The combiner ring [13] will be constructed in 2006 and commissioned in 2006/07 in a group effort of INFN-LNF, CIEMAT, BINP and CERN, with a part of the magnets provided by the decommissioned LURE/Super-ACO facility. This allows to complete demonstration of ILCTRC R1.2 (validation of drive beam generation) in 2007, if operation with nominal parameters can be established by this time. In 2006 the new building for the CLIC experimental area (CLEX) will be constructed. This building will accommodate the probe beam injector, the two-beam test stand and the test beam line (TBL). The two-beam test stand will be used from 2007 on to test PETS [13] with nominal power and on/off features $R 1.3$ and from 2008 on for experiments with a linac module $R 2.3$ consisting of one PETS on the drive-beam connected to four accelerating structures acting on the probe beam.

The probe beam injector is designed and built by a collaboration of CEA-DAPNIA and IN2P3-LAL [14]. It will be put in operation in 2008. The probe beam will not only be used to verify acceleration in the $30 \mathrm{GHz}$ structures, but also to measure effects of RF break-downs, wakefields and beam loading. In order to achieve high measurement resolution a small emittance, very short bunch length $\left(\sigma_{\mathrm{t}}<0.75 \mathrm{ps}\right)$ and the possibility to operate either with single bunches or trains of bunches are required. This will be achieved by the use of an RF photoinjector and velocity bunching [15]. These features make the probe beam also an ideal tool for beam-diagnostic developments.

The Test Beam Line (TBL) is a scaled model of a CLIC decelerator sector. It consists of a well instrumented string of 14 PETS structures embedded in a FODO quadrupole lattice. It will be used to validate beam stability in the drive-beam decelerator as well as the design of an appropriate machine protection system as required by ILC-TRC $R 2.2$. Design issues for TBL are discussed in [16].

\section{NEW PARAMETER SET}

During the last years substantial progress has been made in the understanding of field and pulse length limitations in normal conducting accelerating structures. A large amount of data has been provided by the X-band tests in NLCTA and the Ka-band experiments in CTF II. Other studies have addressed problems of material fatigue related to pulsed heating and plasma dynamics of breakdowns [17]. Parallel with these developments the understanding of low emittance generation in damping rings 


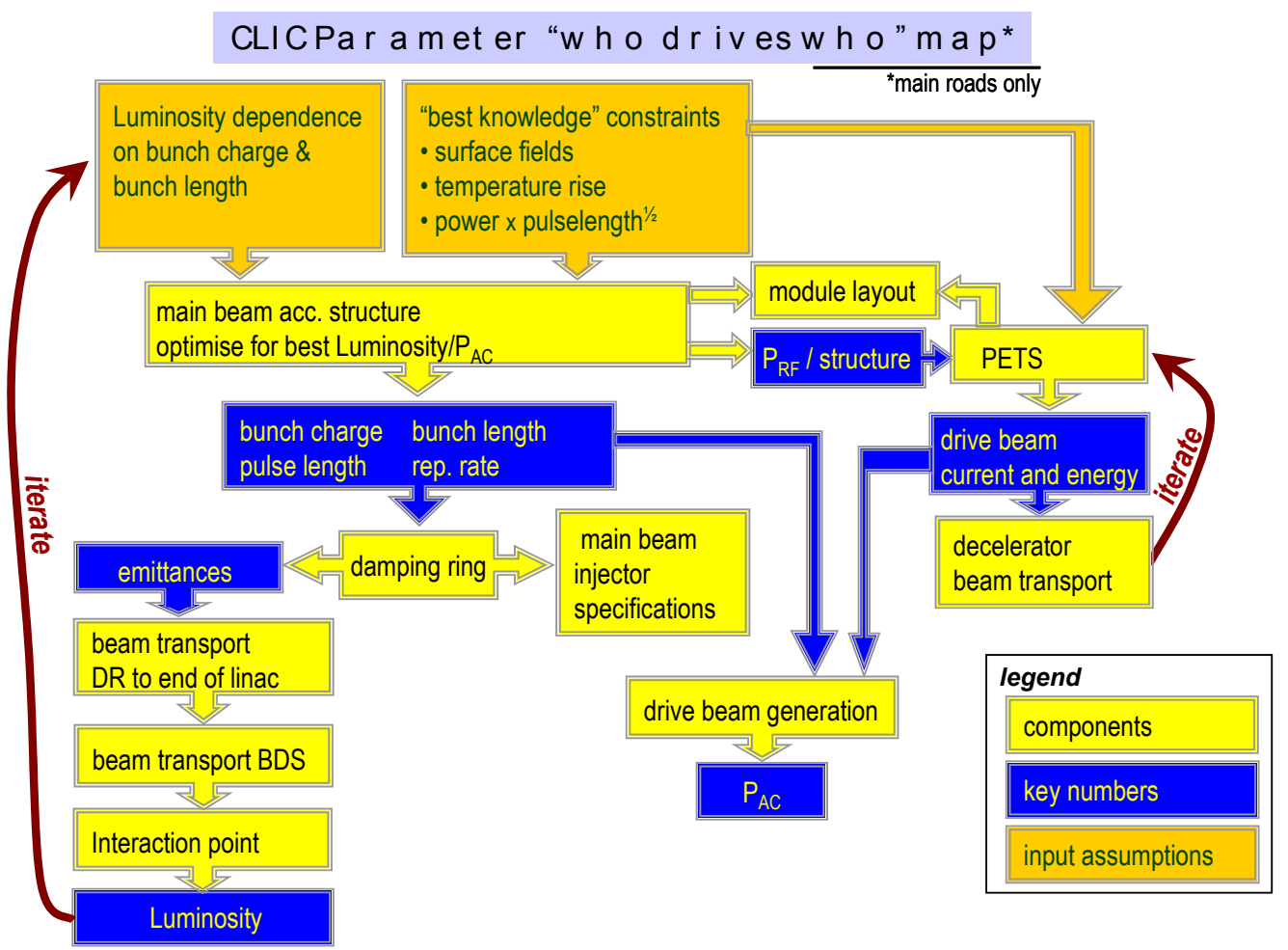

Figure 2: Flow chart of parameter interdependence.

and emittance preservation during beam transport through main linac and beam delivery system has improved and the dependency of luminosity on beam parameters has been studied in detail [18].

In the light of these new results it became apparent that the old CLIC main beam accelerating structure design TDS [19] is not able to operate with the parameters as assumed in [20]. This triggered the development of a new accelerating structure concept named HDS [21]. Although no conclusive theory of RF breakdowns with predictive power exists to date, extrapolations from available data are used for HDS as design guidelines, imposing limits on surface field strengths, power flow and pulse length. As an amalgam from [22,23,24] we adopted, with some safety margins, the following limits for the design of structures build in copper-molybdenum hybrid technology:

$$
\begin{array}{llrl}
\text { - } & \text { Surface electric field } & \hat{E}_{\text {SURF }} & <380 \mathrm{MV} / \mathrm{m} \\
\text { - } & \text { Pulsed surface heating } & \Delta \hat{T}_{S U R F} & <56 \mathrm{~K} \\
\text { - } & \text { Power flow } & P \sqrt{t_{P U L S}} & <1200 \mathrm{MW} \mathrm{ns}^{1 / 2}
\end{array}
$$

While the HDS features a significantly improved damping of long range wake-potentials, the requirements on surface field and power flow forced a reduction in aperture. The unavoidable consequence is an increased short range wake-potential. In order to keep the emittance growth in the main linac under control this necessitates a reduction of bunch charge, while the improved damping allows decreasing the distance between bunches. Although the HDS design has significantly reduced surface fields compared with the TDS the pulsed surface heating for a RF pulse length of $130 \mathrm{~ns}$ as assumed in the old parameters was still unacceptable. A reduction of RF pulse length by roughly a factor of two is therefore needed.

Taking all this into account necessitates a major review and adaptation of the CLIC parameters and subsystem designs to bring the parameters into line with the recent developments. As a specification given by physics requirements [1] we assume that the Luminosity in a $1 \%$ energy bin remains at $3.3 \cdot 10^{34} \mathrm{~cm}^{-2} \mathrm{~s}^{-1}$ and that the center of mass energy is $3 \mathrm{TeV}$. In order to limit the number of dimensions in parameter space we kept some key parameters as fixed input specifications, although further studies may lead to a modification of these parameters as well. The fixed parameters are the loaded accelerating gradient of $150 \mathrm{MV} / \mathrm{m}$ and the main linac RF frequency of $30 \mathrm{GHz}$.

The figure of merit used in the optimization is the Luminosity in a $1 \%$ energy bin divided by the mains power. This was chosen because it facilitates the optimization process compared with a more refined criteria like luminosity divided by total cost (=investment and running costs over some assumed lifetime of the collider). Fig. 2 shows the schematics of the process used to derive the parameters. The necessity to adapt the accelerating structure is the main driving force of the parameter changes compared with the old set of parameters. Nevertheless substantial changes have been applied to many subsystems, in particular the damping rings, the power extraction and transfer structures (PETS) and the drive beam generation complex. These changes are motivated by progress on the system design as well as 
the modified beam characteristics determined from the accelerating structure.

The design of the damping ring and understanding of limiting factors has been improved $[25,26]$. Moreover, the reduction of bunch charge imposed by the accelerating structure design helps to reduce emittance growth due to intrabeam scattering. Recently a new collaboration with the Budker Institute in Novosibirsk (BINP) has started on all aspects of the wiggler design, synchrotron radiation absorption and wiggler beam dynamics [27]. The total wiggler length in the CLIC DR ring is $152 \mathrm{~m}$ for a total circumference of $360 \mathrm{~m}$. A first but important outcome of this collaboration is the reduction of wiggler period length from $20 \mathrm{~cm}$ to $10 \mathrm{~cm}$, leading to a reduction of equilibrium emittances by $20 \%$.

As a consequence of the reduction of bunch spacing in the main linacs from $20 \mathrm{~cm}$ to $8 \mathrm{~cm}$ a train combination system was introduced after the $9 \mathrm{GeV}$ booster linac. This allows keeping the bunch spacing in the damping ring and the injectors at a more conservative value of $16 \mathrm{~cm}$.

The drive beam generation had to be adapted for the new parameters. In particular the shortening of the pulse length required significant modifications, relative to the scheme described in [28]. A single drive beam linac feeds the decelerators of both, the $\mathrm{e}^{-}$and $\mathrm{e}^{+}$main linac. The two combiner rings accumulate two drive beam trains simultaneously instead of one as it was done in the old scheme. This allows to keep the circumference of the rings similar to the old values, despite of the much shorter pulse length.

The PETS design [29] has not only been modified for the new drive beam parameters and HDS power requirements but includes now also the on/off mechanism requested by ILC-TRC. Moreover, a slight variation of RF phase advance along the PETS structure has been introduced to detune high frequency dipole bands. This detuning improves the stability in the decelerator.

Table 1 summarizes the key parameters in comparison with the old values. A complete list of the new parameters together with a detailed description of the design changes and the rational for the parameter changes can be found in [30]. Figure 3 shows the layout and dimensions of CLIC with updated parameters.

One shortfall of the parameters we converged to should be kept in mind. The optimization as defined in [31] leading to a luminosity $L \sim P_{B E A M} \sigma_{Z}^{-1 / 2}$ would require a horizontal IP spot size $\sigma_{H}{ }^{*}$ considerably smaller than the present value of $60 \mathrm{~nm}$ [32]. Substantial progress on beam delivery system design, low emittance generation and emittance preservation is required to achieve this. The benefit would be an increased luminosity to $\mathrm{AC}$ power ratio.

\section{CONCLUSIONS}

The experimental programme of the CTF3 collaboration has been realigned to demonstrate all CLIC key feasibility issues identified by ILC-TRC before 2010.

A major update of CLIC design and parameters has been undertaken. The new parameters comply with present knowledge on RF breakdown limits. The RF to beam power efficiency has been increased and IP backgrounds have been reduced, while effective luminosity, total power consumption and total length remained almost constant

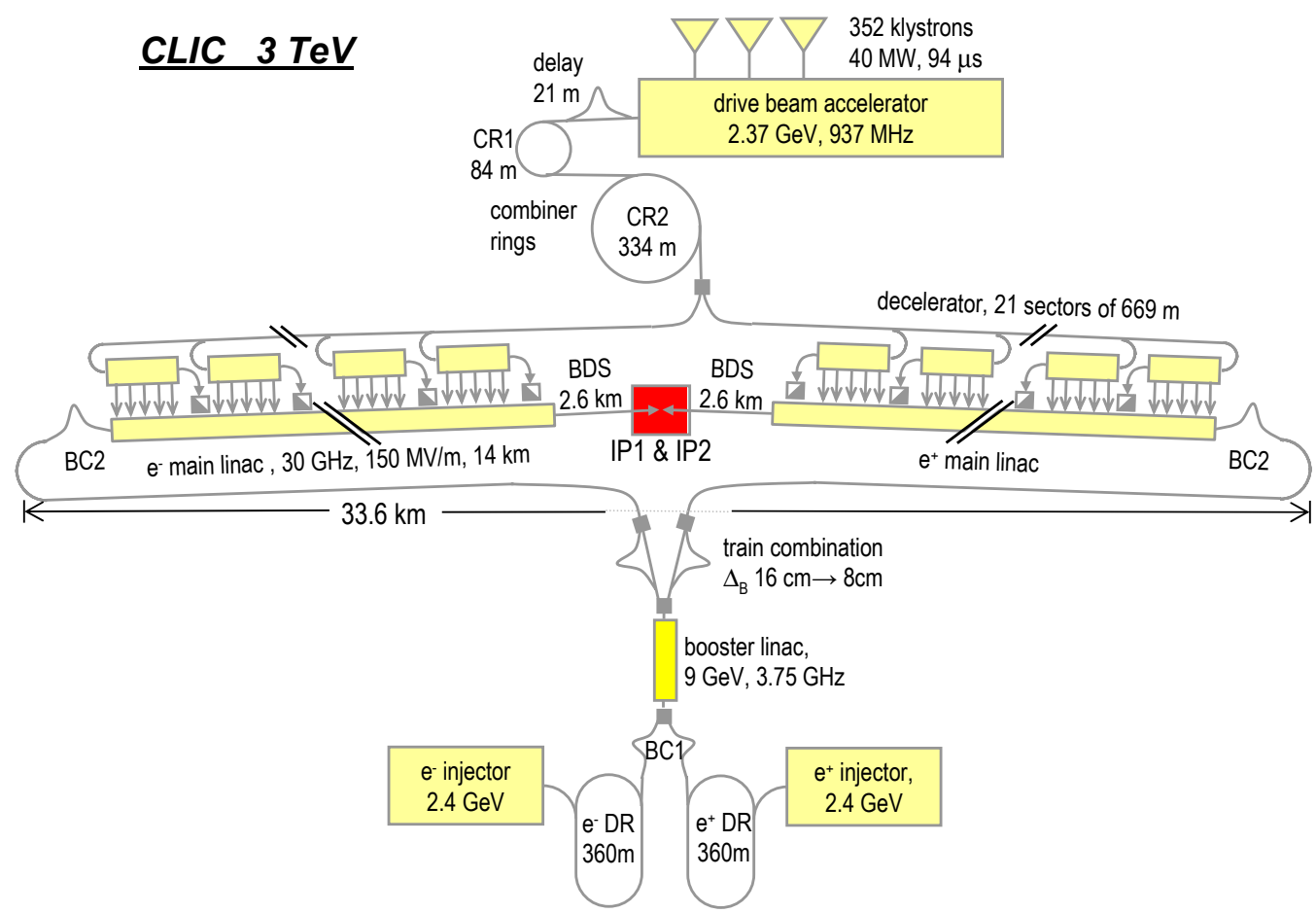

Figure 3: Layout and dimensions of CLIC with updated parameter 
Table 1: comparison of old and new key CLIC parameters

\begin{tabular}{|c|c|c|c|}
\hline Parameter & Unit & old & new \\
\hline $\begin{array}{l}\text { Center of mass } \\
\text { energy }\end{array}$ & $\mathrm{GeV}$ & 3000 & 3000 \\
\hline $\begin{array}{l}\text { Main Linac RF } \\
\text { Frequency }\end{array}$ & $\mathrm{GHz}$ & 30 & 30 \\
\hline $\begin{array}{l}\text { Unloaded / loaded } \\
\text { gradient }\end{array}$ & $\mathrm{MV} / \mathrm{m}$ & $172 / 150$ & $172 / 150$ \\
\hline Linac repetition rate & $\mathrm{Hz}$ & 100 & 150 \\
\hline $\begin{array}{l}\text { No. of particles / } \\
\text { bunch }\end{array}$ & $10^{9}$ & 4.2 & 2.56 \\
\hline $\begin{array}{l}\text { No. of bunches / } \\
\text { pulse }\end{array}$ & & 154 & 220 \\
\hline Bunch separation & ns & 0.67 & 0.267 \\
\hline Bunch train length & ns & 101 & 58.4 \\
\hline Total length & $\mathrm{km}$ & 33.2 & 33.6 \\
\hline Total site AC power & MW & 410 & 418 \\
\hline$\eta_{\mathrm{AC} \text { to main beam power }}$ & $\%$ & 9.3 & 12.5 \\
\hline Luminosity & $\begin{array}{c}10^{34} \\
\mathrm{~cm}^{-2} \mathrm{~s}^{-1}\end{array}$ & 8 & 6.5 \\
\hline $\begin{array}{l}\text { Luminosity } \\
\text { (in } 1 \% \text { of energy) }\end{array}$ & $\begin{array}{c}10^{34} \\
\mathrm{~cm}^{-2} \mathrm{~s}^{-1} \\
\end{array}$ & 3.3 & 3.3 \\
\hline $\begin{array}{l}\text { Beamstrahlung } \\
\text { mom. spread }\end{array}$ & $\%$ & 21.1 & 16 \\
\hline $\begin{array}{l}\sigma_{\mathrm{H}}{ }^{*} / \sigma_{\mathrm{V}}{ }^{*} \\
\text { before pinch }\end{array}$ & $\mathrm{nm}$ & $60 / 0.7$ & $60 / 0.7$ \\
\hline
\end{tabular}

\section{REFERENCES}

[1] M. Battaglia, A. de Roeck, J. Ellis, D. Schulte (editors), "Physics at the CLIC Multi-TeV Linear Collider, rep. of the CLIC Physics Working Group," CERN report 2004-005

[2] R. Corsini for the CLIC Study Team, " Overview of CLIC and CTF3," proc. LINAC 2002 and CLIC note 540

[3] H.H. Braun for the CLIC study team, "Achievements and Future Plans of CLIC Test Facilities," proc. HEACC 2001 and CLIC note 473

[4] "International Linear Collider Technical Review Committee, $2^{\text {nd }}$ report," SLAC Report 606, 2003

[5] I. Wilson for the CLIC study team, "CLIC Accelerated R\&D," CLIC note 620, 2005

[6] E. Jensen, P. Pearce, I. Syratchev, "A Novel Idea for a CLIC 937 MHz 50 MW Multibeam Klystron," CLIC note 590, 2004

[7] "European Design Study towards a Global TeV Linear Collider," http://www.eurotev.org

[8] R. Corsini et al., "First Full Beam Loading Operation with the CTF3 Linac", Proc. EPAC'04 and CLIC note 604,2004

[9] R. Corsini, presentation at "Ninth CLIC/CTF3 Collaboration Meeting," CLIC note 619, 2004

[10] T. Lefèvre, M. Velasco, M. Wood, H.H. Braun, R. Corsini, M. Gasior, "Beam Loss Monitoring at the CTF3”, proc. EPAC'04 and CLIC note 611, 2004
[11] A. Ghigo et al. , "Commissioning and First Measurements on the CTF3 Chicane," this conf.

[12] W. Wuensch et al., "30 GHz Power Production in CTF3," this conf.

[13] C. Biscari et al., "CTF3: Design of Drive Beam Combiner Ring," Proc. EPAC 2000

[14] M. Jablonka, presentation at "Ninth CLIC/CTF3 Collaboration Meeting," CLIC note 619, 2004

[15] L. Serafini, A. Bacci, M. Ferrario, "Ultra-Short Electron Bunch Generation with a Rectilinear Compressor," Proc. PAC 2001

[16]D. Schulte, I. Syratchev, "Considerations on the Design of the Decelerator of CTF3," this conf.

[17] Presentations at "Workshop on High Gradient RF", ANL 2003, http://www.mice.iit.edu/rfworkshop/

[18]D. Schulte, "Luminosity Limitations at the Multi TeV Linear Collider Energy Frontier," Proc. EPAC 2002 and CLIC note 527, 2002

[19] J.Y. Raguin, I.Wilson, W.Wuensch, "Progress in the Design of a Damped and Tapered Accelerating Structure for CLIC', Proc. PAC'03 and CLIC note 567,2003

[20] G. Guignard (editor), "CLIC Contribution to the Technical Review Committee on a $500 \mathrm{GeV} \mathrm{e}^{+} / \mathrm{e}^{-}$ Linear Collider", CERN report 2003-7

[21] A. Grudiev, W. Wuensch, “A Newly Designed and Optimized CLIC Main Linac Accelerating Structure", Proc. LINAC'04 and CLIC note 601, 2004

[22]W. Wuensch et al., "A Demonstration of HighGradient Acceleration", PAC'03 and CLIC note 569, 2003.

[23] S. Heikkinen, http://cern.ch/clic-meeting/, CLIC Meeting of 26 June 2003.

[24] V. Dolgashev, "Effect of RF Magnetic Fields and Input Power on RF Breakdown Limit", see [17]

[25] M. Korostelev, F. Zimmermann, “CLIC Damping Ring Optics Design Studies,” this conf.

[26]F. Zimmermann, M. Korostelev, D. Schulte (CERN), T. Agoh, K. Yokoya (KEK), "Collective Effects in the CLIC Damping Rings," this conf.

[27] E.B. Levichev, P.D. Vobly, P.A. Piminov and K.V. Zolotarev, "CLIC Damping Ring Wigglers: Magnet Design, Beam Dynamics and SR Absorbers," CLIC note in preparation

[28] R. Corsini (ed.),'The CLIC RF Power Source: a Novel Scheme of Two-Beam Acceleration for Electron-Positron Linear Colliders", CERN report 99-06 (1999).

[29] I. Syratchev, presentation at "Ninth CLIC/CTF3 Collaboration Meeting," CLIC note 619, 2004

[30] F. Tecker (ed.), "Updated CLIC parameters 2005," CLIC note 627, 2005

[31] J.P. Delahaye, G. Guignard, T. Raubenheimer and I.Wilson, "Scaling Laws for $\mathrm{e}^{+} / \mathrm{e}^{-}$Linear Colliders," CLIC note 333, 1997

[32] H.H. Braun, D. Schulte, "Optimum Choice of RF Frequency for Two Beam Linear Colliders", proc. PAC'03 and CLIC note 563, 2003 